\title{
Applications of an Airborne Ruby Lidar During a BOMEX Program of Cirrus Observations ${ }^{1}$
}

\author{
Paul A. Davis \\ Stanford Research Institule, Menlo Park, Calif. \\ (Manuscript received 26 January 1971, in revised form 16 September 1971)
}

\begin{abstract}
A ruby lidar was mounted in an aircraft to obtain measurements of cirrus and haze layers concurrently with an airborne infrared radiometer during Project BOMEX in July 1969. The capability of the lidar for detecting cirrus or haze layers and for describing directly their range and thickness provided uniquely the information essential to a meaningful analysis of radiometric data. In addition to the geometric descriptions, analyses of lidar returns were performed to determine optical parameters. The procedure for conducting these analyses required that an average profile of the clear air backscattering coefficient in the troposphere above the boundary layer be established. Samples of lidar data and derived optical parameters are shown; one illustration includes data from a dense dust layer below an altitude of $12,000 \mathrm{ft}$.

Comparison of analyses of lidar and radiometric data from cirrus clouds indicated that the infrared transmittance $(10.2-11.6 \mu)$ was directly related to the geometric thickness of the cloud. This important statistical result suggests a basis for simplified modeling of the influence of cirrus on infrared transfer.
\end{abstract}

\section{Introduction and objectives}

An uncertainty exists with respect to the radiative influence of cirrus or haze layers within the real atmosphere. Strictly theoretical determinations of the effects of particulates on radiative transfer require information on particle sizes, shapes, refractive indices, and concentrations, as well as their spatial variability. On the other hand, attempts to infer gross radiative properties of transmittance and reflectance for particulate layers from empirical (radiometric) measurements are hampered by the lack of specific information on the presence, range and thickness of these layers or other layers in the background. However, if a lidar system is mounted so as to provide concurrent measurements with the radiometer, the presence, range and thickness of tenuous cirrus or haze layers may be defined uniquely. Such definition is essential to a meaningful analysis of the radiometric data.

During the July phase of the 1969 Barbados Oceanographic and Meteorological Experiment (BOMEX), a ruby laser system was installed aboard the NASA CV-990 aircraft to obtain coordinated lidar measurements of cirrus and haze with measurements ${ }^{2}$ by a narrow-aperture chopper-bolometer infrared radiometer. The basic objective was to seek a relationship between the lidar signatures and the IR transmittances deduced from radiance measurements in the 10.2 to $11.6 \mu$ region

\footnotetext{
'This work is based on research sponsored by the National Environmental Satellite Service, NOAA, under Contract E-263-68. ${ }^{2}$ Conducted by Peter M. Kuhn, Environmental Research Laboratories, NOAA.
}

of the spectrum and from temperature data. In addition to the description of geometric properties of measurable cirrus clouds, quantitative analyses were performed on many of the lidar traces in order to derive optical thickness and total transmittance at the ruby wavelength $(0.6943 \mu)$ along with profiles of the volume backscattering coefficient. These optical properties provide an objective means for the identification of potential differences in independent particulate layers with either the same or different geometrical properties.

The installation of the lidar with the radiometer in the aircraft made it possible to realize certain advantages not available from ground-based operation. Most important to this study was the ease with which cirrus layers could be encountered on any given flight. Radiance measurements were enhanced by the elimination of emitting and absorbing portions of the intervening atmosphere and by obtaining radiometric and temperature measurements at cloud boundaries or within clouds. For daytime operation of the lidar the principal advantages of the airborne operation were twofold: a significant gain in signal amplitude at the target by virtue of the reduction in range, and a reduction in the daytime background light level. Clear air returns with magnitudes exceeding the background provided the basis for referencing returns from cirrus clouds.

\section{Lidar data samples}

Details of the operational airborne lidar system have been discussed previously (Davis, 1970). Table 1 
summarizes the basic characteristics of the optical and data-recording components.

Fig. 1 shows Polaroid photographs of oscilloscope traces swept from left to right at a preset writing speed. The amplitude of the oscilloscope deflection (denoted by the ordinate $A$ ) is proportional to the logarithm of the received power. The trace in Fig. 1a was recorded at a low altitude over the BOMEX area, with the lidar directed upward. The heavy solid line near the top of the grid represents the data baseline; the baseline was recorded only occasionally. The small initial spot at the left of the trace near the baseline represents the daytime background light level entering the receiver system. The number of scatterers and the angle at which they are viewed relative to the sun determine the magnitude of the background. As the receiver field of view encompasses most of the transmitted pulse, the amplitude of the return (increasing downward on the photograph) reaches a maximum at a range (increasing to the right) of $\sim 200 \mathrm{~m}$, but full convergence of the transmitter and receiver beams for most settings of the receiver was not achieved until a range of $\sim 400 \mathrm{~m}$. As the range increases further, the trace of the signal backscattered from the cloud-free air declines approximately as the reciprocal as the square of the range until the background level is reached, and then remains at that level.

Fig. 1b shows the sharp increase in the backscattered signal as the downward transmitted pulse enters a small water cloud. On the far side of the cloud the return trace from the cloud-free air is reduced (offset), as a result of cloud attenuation, from the magnitude that would have been apparent in the absence of the cloud. Fig. 1c illustrates a view down to the sea surface from about an 18,000-ft altitude and a $30^{\circ}$ nadir angle. The background light level is extremely low. As the laser pulse reaches the sea surface, a sharp narrow reflection spike

TABLE 1. Lidar system aboard NASA CV-990 aircraft.

\begin{tabular}{|c|c|}
\hline \multicolumn{2}{|l|}{ Transmitter } \\
\hline $\begin{array}{l}\text { Wavelength } \\
\text { Laser } \\
Q \text {-switch } \\
\text { Optics } \\
\text { Pulse duration } \\
\text { Energy output } \\
\text { Beamwidth }\end{array}$ & $\begin{array}{l}6943 \AA \\
\frac{3}{8} \text {-inch ruby rod, Brewster-angle one end } \\
\text { rotating prism and saturable dye } \\
\text { expanding and } 2 \text {-inch collimating telescope } \\
20 \text {-nsec minimum } \\
0.25-0.5 \mathrm{~J} \\
\text { approximately } 0.5 \mathrm{mrad}\end{array}$ \\
\hline \multicolumn{2}{|l|}{ Receiver } \\
\hline $\begin{array}{l}\text { Optics } \\
\text { Field of view } \\
\text { Filter bandpass } \\
\text { Photomultiplier } \\
\text { Display mode }\end{array}$ & $\begin{array}{l}\text { 6-inch Newtonian reflector } \\
\text { adjustable, 0.5-5.0 mrad } \\
13 \AA \\
\text { RCA } 7265 \text { (S-20 cathode) } \\
\text { either linear or logarithmic amplification } \\
\text { (dc coupled) }\end{array}$ \\
\hline \multicolumn{2}{|l|}{ Recording } \\
\hline \multicolumn{2}{|c|}{$\begin{array}{l}\text { Polaroid photograph of TEK } 454 \text { oscilloscope display } \\
35 \text {-mm film (automatic camera) of TEK } 453 \text { oscilloscope display } \\
\text { Strip-chart monitor of transmitted peak power or energy } \\
\text { Audio tape recorder } \\
\text { Housekeeping information for aircraft (provided by NASA) }\end{array}$} \\
\hline
\end{tabular}
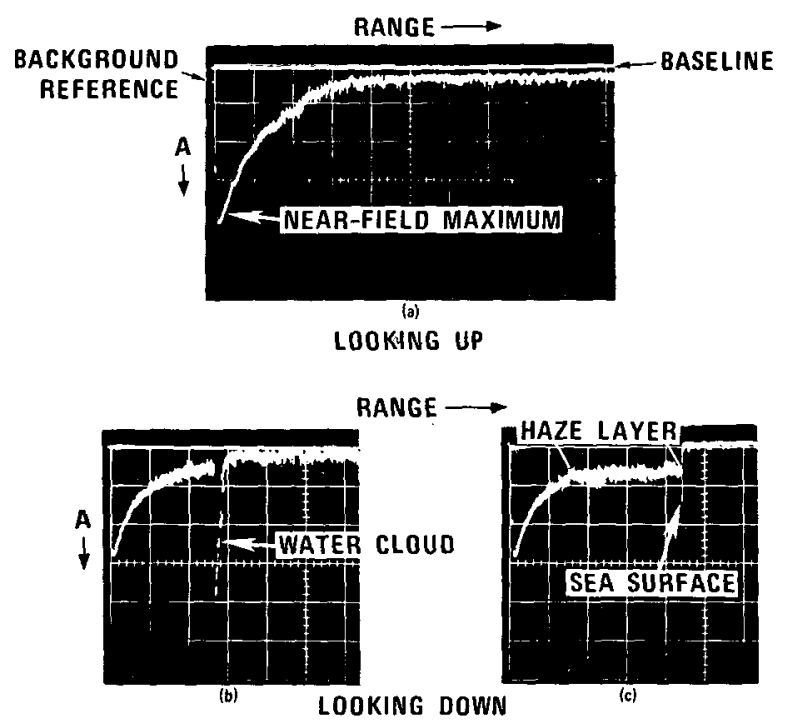

FIG. 1. Sample oscilloscope traces of lidar returns, where the deflections are proportional to the logarithm of the received power.

is apparent, followed by an abrupt decline of the signal to the background level. The magnitude of the reflection spike, which is not always detectable, depends on the angle of incidence of the pulse, the transmitted power, the two-way attenuation between the lidar and the sea surface, the roughness of the sea surface, and the turbidity of the surface water. Well before the sea surface is reached the return signal ceases to decline inversely with the square of the range; this portion of the trace depicts the increased backscattering as the boundary haze layer is penetrated.

Fig. 2a shows the oscilloscope trace for an upward view from the aircraft at an altitude of $33,000 \mathrm{ft}$, with a tenuous double layer of cirrus (about $2500 \mathrm{ft}$ thick) based some $3500 \mathrm{ft}$ above the aircraft. The return trace from the clear air on the far side of the cloud is not noticeably different from the return from the same range

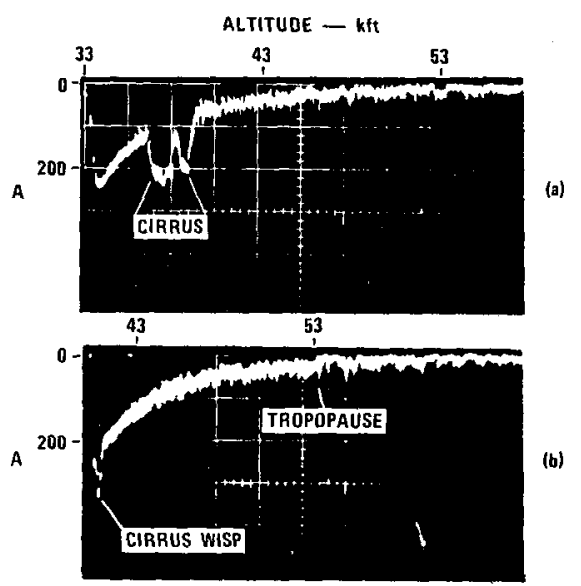

Fig. 2. Oscilloscope traces for upward-directed lidar shots, 23 July 1969. 
in the absence of the cloud. This trace illustrates the difficulty in estimating directly the total cirrus transmittance even when the analog records on both sides of the cloud are significantly above the background. Fig. $2 b$ shows the received signal from an upward view at an aircraft altitude of $40,000 \mathrm{ft}$. At the instant of this record a very thin cirrus wisp passed in front of the lidar (note the near-field return). At a range corresponding to an altitude of $\sim 53,000 \mathrm{ft}$, the backscattered signal shows a decline to a lower magnitude at approximately the background level. On this day the tropopause was also observed at about $53,000 \mathrm{ft}$. At higher altitudes the intensity of the backscattered signal can only be assessed as a function of range by comparing the average number of photoelectrons released from the cathode of the photomultiplier when a pulse is transmitted with the background count of photoelectrons when no energy is transmitted. At night the background count frequently can be neglected (cf. Viezee et al., 1971).

Fig. 3 presents samples from a series of traces from upward-directed lidar shots during descent of the aircraft from 37,000 to $4000 \mathrm{ft}$ in the clear air below a cirrus deck at $\sim 40,000 \mathrm{ft}$. Although the amplitude of the return signal from a fixed target decreases with increasing range, the weak cirrus returns observed from and below $10,000 \mathrm{ft}$ resulted primarily from the much thinner cirrus cover at those positions of the aircraft. As the aircraft descends into the lower atmosphere, the backscattered signal from the cloud-free air increases as a result of the increasing density of particulates and molecules. The increase in the backscattering is most noticeable in the amplitude of the signal maximum at

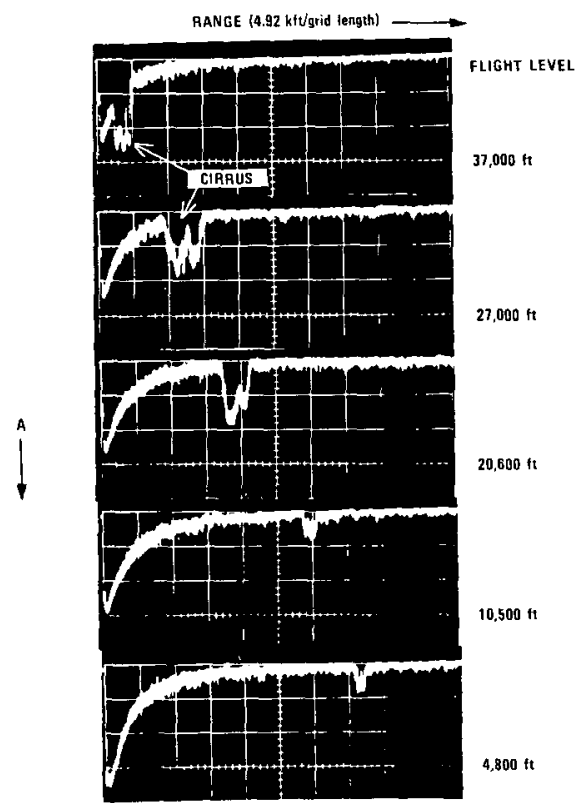

FIG. 3. Upward-directed lidar returns during descent below cirrus clouds, 22 July 1969. close range, where the signal is well above the background. An analysis of the large-amplitude near-field returns during ascents or descents gives a reliable description of an average relative backscattering profile for clear air at all altitudes traversed by the aircraft. From the ground, instantaneous analog profiles of the daytime clear air backscattering through the upper troposphere could only be acquired with a high performance lidar of considerably greater sophistication than that used in the aircraft.

It is apparent from these data samples that the detection and geometric ranging of particulate layers is easily accomplished by an analysis of the return trace. A completely quantitative analysis of lidar returns in terms of the volume backscattering coefficient (corrected for attenuation) and the optical thickness is much more difficult but can be accomplished in a meaningful approximate manner.

\section{The lidar equation and analyses for optical parameters}

A basic technique for the approximate solution of the lidar equation and an analysis in terms of profiles of the volume backscattering coefficient, the optical thickness, and the transmittance through a cirrus layer has been described (Davis, 1969). With minor alterations the same approach has been used here.

The lidar equation can be expressed in the form

$$
\operatorname{Pr}^{2}=f E_{t} \beta_{\pi} T^{2}{ }_{o, r}
$$

where $P$, in watts, is the power received from range $r, f$ a constant equal to one-half the product of speed of light, receiver aperture area, and efficiency factor for the system $\left(\mathrm{km}^{3} \mathrm{sec}^{-1}\right), E_{t}$ the transmitted energy $(\mathrm{J})$, $\beta_{\pi}$ the volume backscattering coefficient $\left(\mathrm{km}^{-1} \mathrm{sr}^{-1}\right)$ at range $r$, and $T_{0, r}$ the transmittance over the path between the lidar and range $r$. In practice, the two-way transmittance between any two range points, $x, y$, is represented by

$$
T^{2}{ }_{x, y}=\exp \left(-2 \int_{x}^{y} \gamma d r\right)
$$

where $\gamma$ is the apparent extinction coefficient $\left(\mathrm{km}^{-1}\right)$ at range $r$ and the integral represents the optical thickness between $x$ and $y$.

Both the background power of ambient light entering the receiver and the total power received during a lidar firing are measured as oscilloscope deflections and calibrated in terms of relative light input to the receiver. From both measurements the relative light input due to backscattering from the lidar pulse alone can be determined. If $I(r)$ and $I(R)$ are, respectively, the relative light inputs (proportional to the logarithms of the received powers) from an arbitrary range $r$ and a reference range $R$ just inside the cloud boundary, then the lidar equation can be expressed in terms of the 
range-corrected signal ratio $S$ at range $r$ :

$$
\begin{aligned}
S(r) \equiv I(r)-I(R) & +\ln (r / R)^{2} \\
& =\ln \left[\beta_{\pi}(r) / \beta_{\pi}(R)\right]-2 \int_{R}^{r} \gamma(r) d r,
\end{aligned}
$$

where the integral represents the optical thickness $D(r)$. A relationship between $\gamma$ and $\beta_{\pi}$ is assumed in the form

$$
\gamma(r)=b C(r) \beta_{\pi}(r)
$$

where $b$ is treated as a constant for a given medium and $C(r)$ is an empirically determined function of optical thickness or range. Within a reasonably uniform medium $C(r)$ is assumed to be unity if the multiple scattering of the transmitted pulse into the forward direction is insignificant over the viewed optical path.

With (4) the solution of (3) for $\beta_{\pi}$ becomes

$$
\beta_{\pi}(r)=e^{S(r)}\left[\frac{1}{\beta_{\pi}(R)}-2 b \int_{R}^{r} C(h) e^{S(h)} d h\right]^{-1} .
$$

The quantity $S(r)$ is computed directly from a digital record of the lidar trace, or as the average at each range point on a number of digitized traces that have been acquired at a high pulse-repetition frequency. Since $C(h)$ is unity at the initial penetration of the cloud and decreases very slowly with optical thickness in a quasiuniform medium, solutions to (5) are obtained first with $C(h)$ set at unity, but both $\beta_{\pi}(R)$ and $b$ must be specified as input parameters.

To determine $\beta_{\pi}(R)$, it was necessary to establish a clear air reference backscattering coefficient, $\beta_{\pi}\left(L_{z}\right)$, at range $L$ from the lidar for any given altitude $z$. During cloud-free portions of ascents or descents, the data from the airborne lidar can be analyzed directly in terms of the ratio

$$
\begin{array}{r}
\ln \left[\beta_{\pi}\left(L_{z}\right) / \beta_{\pi}\left(L_{d}\right)\right] \\
\quad=I\left(L_{z}\right)-I\left(L_{d}\right)+\operatorname{In}\left[E_{t}\left(L_{d}\right) / E_{l}\left(L_{z}\right)\right],
\end{array}
$$

where the normalization altitude $d$ preferably should be selected at some height for which a minimum variation in particulate backscattering is anticipated. The last term in (6) is zero for points on the same lidar trace but may also be neglected if $I\left(L_{z}\right)$ and $I\left(L_{d}\right)$ are averaged from a number of traces. From the average profile of the relative backscattering coefficient described from (6) as a function of height, the magnitude of $\beta_{\pi}\left(L_{z}\right)$ at any height $z$ is determined once the magnitude of the backscattering coefficient can be specified by independent means for any one point on the profile. Given $\beta_{\pi}\left(L_{z}\right)$, the reciprocal of $\beta_{\pi}(R)$ is computed from

$\frac{1}{\beta_{\pi}(R)}=\exp (-2 \bar{\gamma} R) \frac{E_{t}(R)}{E_{t}(L)}\left(\frac{L}{R}\right)^{2} \frac{\exp [I(L)-I(R)]}{\beta_{\pi}\left(L_{2}\right)}$,

where $\bar{\gamma}$ is the average extinction coefficient between the lidar and range $R$. If the lidar shot is taken from within the cloud, $\vec{\gamma}$ is estimated by an iterative procedure; otherwise, the exponential term containing $\tilde{\gamma}$ is normally treated as unity.

Once the reciprocal of $\beta_{\pi}(R)$ is specified, the solution of $(5)$ is carried out for a variety of magnitudes of $b$ [with $C(h)$ initially set at unity]. Along with each computation of $\beta_{\pi}(r)$ the optical thickness $D(r)$ is computed with the aid of (3); the term $\exp [-D(r)]$ gives the accumulative transmittance at the ruby wavelength. Resultant total transmittances are subsequently compared with total transmittances inferred directly from select lidar traces themselves (e.g., by the decline in the amplitude of the analog signal from the far side of the cloud with respect to the amplitude at the same range in clear skies, or by the selection of a return from an optical thickness sufficiently large to result in nearly complete attenuation of the lidar pulse). The appropriate magnitude of $b$ for the solution of (5) is determined by the most favorable comparison between computed and inferred total transmittances. If a definite decrease of $b$ with increasing optical thickness is established, then the largest magnitude of $b$ is retained and an appropriate $C(h)$ is introduced in the simplified form

$$
C(r)=1-k(r-R),
$$

where $k$ is a constant, and (5) is solved again for new profiles of $\beta_{\pi}(r)$ and the transmittance.

\section{Samples of data analysis}

Fig. 4 illustrates the relative vertical profile of the backscattering coefficient, normalized to an altitude of $\sim 22,000 \mathrm{ft}$, that was determined in accordance with (6) from lidar observations during ascent and descent of the NASA CV-990 aircraft in clear skies off the California coast on 11 September 1969. During both ascent and descent, which were separated in time by

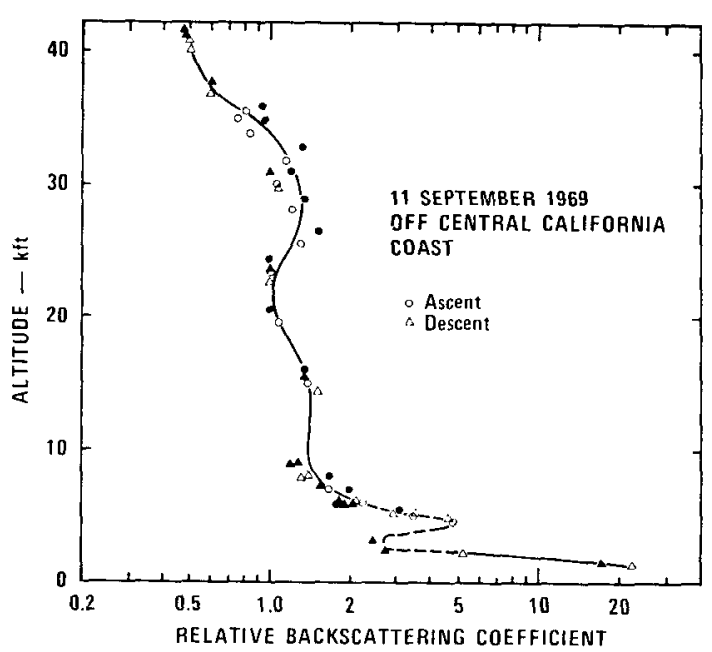

FIG. 4. Profile of the relative backscattering coefficient derived from lidar returns during ascent and descent of aircraft. 


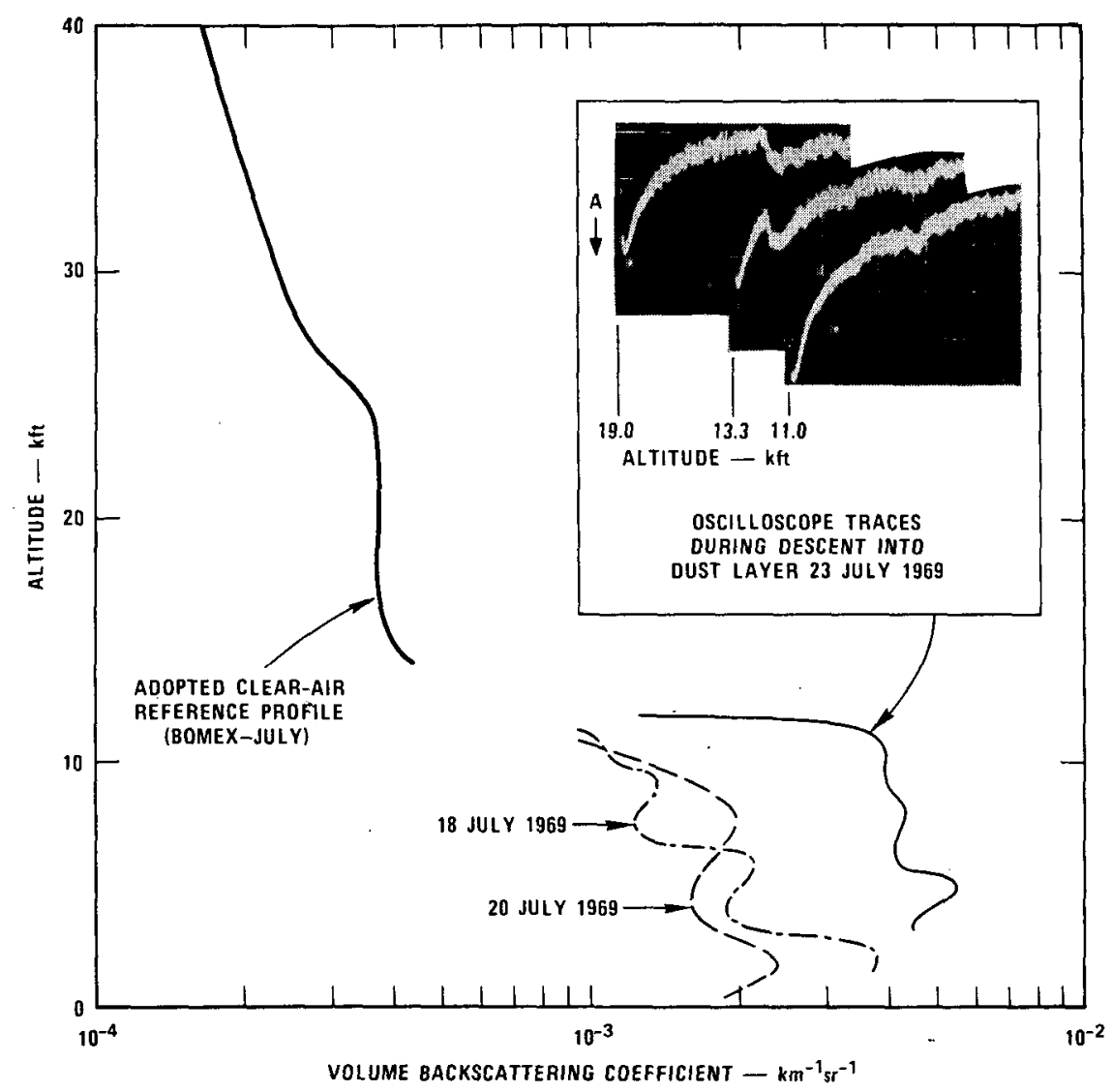

Fig. 5. Average profile of backscattering coefficients at $0.6943 \mu$ deduced from lidar returns in cloud-free air over the BOMEX area in July 1969, and individual boundary layer profiles.

over $4.5 \mathrm{hr}$, Eq. (6) was applied to two different ranges from the lidar. A clear air haze layer is evident between 25,000 and $36,000 \mathrm{ft}$; at one point a tenuous cirrus cloud was observed within the upper portion of this layer. A comparison of the relative minimum in the backscattering near $9000 \mathrm{ft}$, with variations in the backscattering from a Rayleigh atmosphere, indicated that the total backscattering coefficients (at a $180^{\circ}$ scattering angle) near 20,000 and 40,000 ft had to be at least $10 \%$ larger than the molecular backscattering coefficients at the same altitudes.

To derive optical parameters from analyses of lidar observations, it was necessary (as described above) to generate an average profile of the clear air backscattering coefficient from about 15,000 up to $40,000 \mathrm{ft}$. The assignment of absolute magnitudes to the backscattering profile was based on computations of the molecular and particulate backscattering for altitudes at which independent measurements (made aboard the aircraft) of particle size distributions were obtained. Most of the natural variability in particle loading occurs below $13,000 \mathrm{ft}$; this variability has been documented by June 1969 observations with a neodymium lidar

\footnotetext{
${ }^{2}$ Particle counts were obtained by Colorado State University.
}

operating at $1.06 \mu$ (Johnson and Uthe, 1971). Nevertheless, the extremely low particle counts observed at higher altitudes made it necessary to perform the computations at altitudes below $13,000 \mathrm{ft}$ when ruby lidar observations were also available. Results based on the particle counts are discussed in the Appendix.

Fig. 5 illustrates the adopted profile of the backscattering coefficient at $0.6943 \mu$ for clear air. Also shown are three profiles through the boundary layer; each of these profiles was determined from the analysis of a single lidar trace. At 1632 (all times GMT) on 18 July the lidar was directed upward from a flight altitude of about $700 \mathrm{ft}$ at $13 \mathrm{~N}, 54.5 \mathrm{~W}$. The similar profile at 1839 on 20 July was obtained from the return observed at $19,000 \mathrm{ft}$ with the lidar directed downward at $13.5 \mathrm{~N}, 58.3 \mathrm{~W}$. At about 1853 on 23 July the backscattering profile was acquired just before the aircraft descended into a dense dust layer at $12.6 \mathrm{~N}, 59.1 \mathrm{~W}$. Portions of three oscilloscope traces acquired with the lidar directed at a $60^{\circ}$ nadir angle during descent into the dust layer are reproduced in Fig. 5. The extinction coefficient associated with the backscattering profile from the dust layer is about three times larger than the 

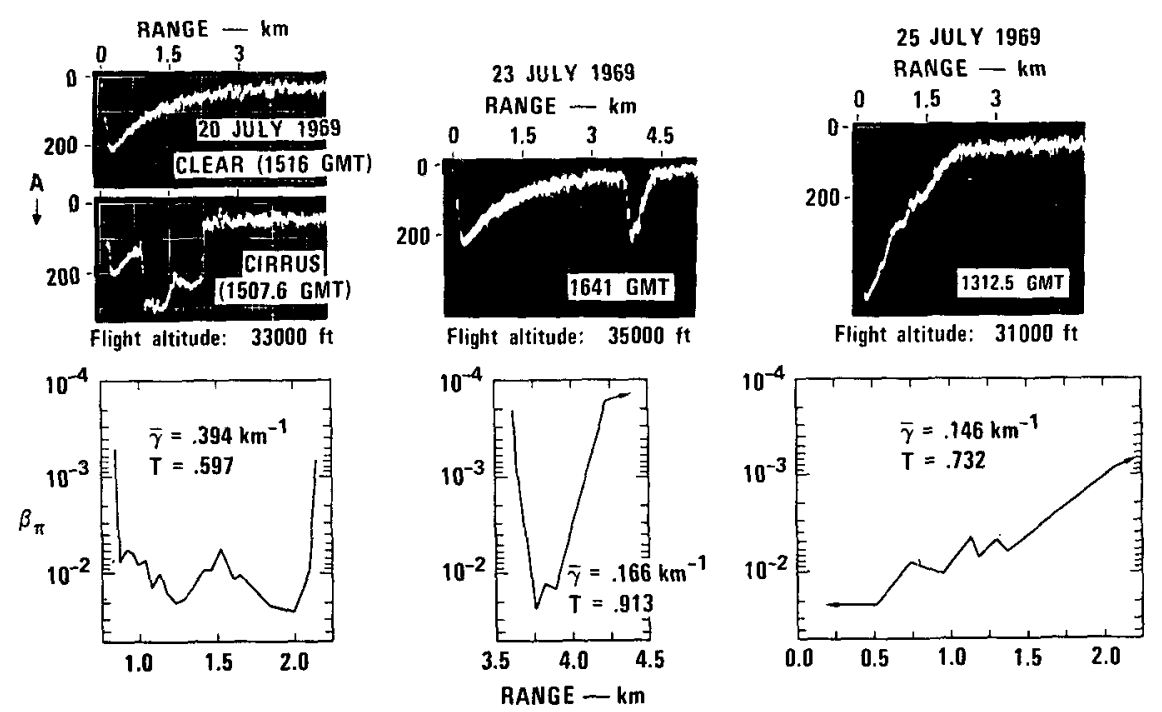

FIG. 6. Sample lidar traces and optical analyses through three cirrus clouds.

extinction coefficients associated with the other two profiles.

With the profile of $\beta_{\pi}\left(L_{z}\right)$ shown in Fig. 5 it was possible to compute profiles of the backscattering coefficient from lidar traces through observed portions of cirrus clouds. Fig. 6 shows the results derived from the analytical analyses of the returns from three upward-directed lidar shots over the BOMEX area. Computed parameters appearing in Fig. 6 are based on the maximum values of $b(32 \mathrm{sr})$ and on a value of $0.09 \mathrm{~km}^{-1}$ for $k$ in Eq. (8). The indicated average extinction coefficients and total transmittances at the ruby wavelength are considered to approximate the upper and lower limits, respectively, for the illustrated returns. The cirrus cloud on 20 July possesses welldefined boundaries and was observed at typical cirrus altitudes. The return for 23 July represents the most substantial, isolated cirrus cloud observed near the tropopause. For 25 July the profile shown in Fig. 6 is the result of a lidar shot through the upper portion of the cirrus cloud from within the cloud. In all three cases the maximum values of the volume backscattering coefficient are approximately the same, but the average extinction coefficient is largest for the cirrus cloud observed on 20 July 1969.

\section{Infrared transmittance of cirrus clouds}

Ideally, an airborne radiometer could obtain upward and downward IR radiance measurements above and below the same cirrus cloud. In addition, upward flux measurements could be made for the same spectral bandwidth as the radiance measurement. These data would provide sufficient information from which the total cloud transmittance could be deduced. However, if the radiometer is directed at a nearly constant background, such as the sea surface or the clear upper atmosphere, it is possible to estimate the transmittance from an aircraft pass at a single altitude from above or below the cloud. Over the BOMEX area it was necessary to attempt the latter procedure.

The following definitions for a given spectral interval are used:

$N \downarrow$ downward radiance measured at cloud base

$Y_{0} \downarrow$ downward radiance measured at cloud base height in absence of cloud

$\Lambda_{+} \downarrow$ downward radiance from atmosphere above cloud

$B_{c} \quad$ Planckian radiance at cloud temperature

$\rho_{c} \quad$ cloud reflectance

$\tau_{c} \quad$ cloud transmittance

$F \uparrow \quad$ upward flux at cloud base

Suppose that the measurement is made sufficiently close to the base of the cloud that the intervening atmosphere can be neglected. If the cloud is treated as an equivalent isothermal sheet, then the measured downward radiance can be defined (Kuhn, 1970) as

$$
N \downarrow \approx \tau_{c} N_{+} \downarrow+\left(1-\tau_{c}-\rho_{c}\right) \bar{B}_{c}+\rho_{c} \frac{F \uparrow}{\pi},
$$

where $\bar{B}_{c}$ is assigned a magnitude for the average cloud temperature (generally this is not a bad approximation). An evaluation of the first two terms of this expression suggest that they lead to slight underestimates. Consequently, a good approximation for the measured downward radiance is

$$
N \downarrow \approx \tau_{c} N_{0} \downarrow+\left(1-\tau_{c}\right) \bar{B}_{c}+\rho_{c}\left(\frac{F \uparrow}{\pi}-\bar{B}_{c}\right) .
$$

A similar expression can be associated with radio- 
metric measurements from downward views on the upper boundary of the cloud, but it is more difficult to separate a generalized multiple-scattering (reflectance) term from the cloud transmittance term.

Solving (9) for the cloud transmittance gives

$$
\tau_{c}=\frac{\left[\bar{B}_{c}-N \downarrow\right]}{\left[\bar{B}_{c}-N_{0} \downarrow\right]}+\frac{\rho_{c}\left[(F \uparrow / \pi)-\bar{B}_{c}\right]}{\left[\bar{B}_{c}-N_{0} \downarrow\right]} .
$$

Some uncertainties in the computed Planckian radiances for the spectral interval arose because of occasional difficulties in obtaining an accurate measure of the ambient air temperature. The general procedure for determining the average temperature of the cloud was to use the lidar signature for a description of the distance and thickness of the cloud and then to extrapolate the ambient temperature upward with a standard lapse rate for the tropical atmosphere. In some regions, especially with extensive cirrus cloud aloft, it was not possible to obtain an immediate measure of downwind radiance
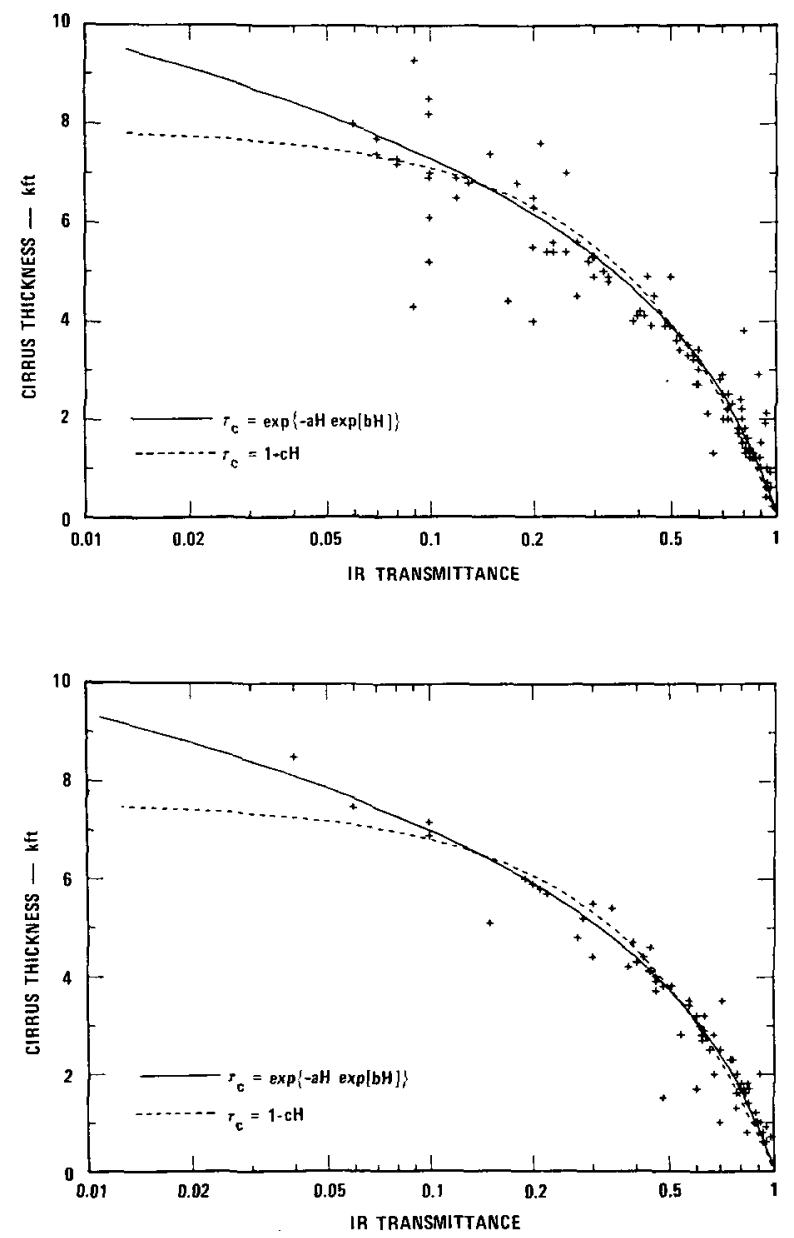

FIG. 7. Infrared transmittance (inferred by neglecting reflectance) and geometrical thickness of cirrus clouds from concurrent radiometric and lidar?views for the same cloud volume (upper) and differing volumes of the same cloud (lower).
$\Lambda_{0} \downarrow$. In other regions the radiometer and lidar were not viewing along the same cloud path or may have been slightly mismatched in time. Thus, the observed radiances and the computed Planckian radiance were subject to variability that introduced scatter when comparisons were made with lidar-derived cloud thicknesses.

A comparison of the deduced IR transmittance to the cirrus cloud thickness was limited by the absence of information on the reflectance parameter $\rho_{c}$. In addition, the spectral flux measurements $F \uparrow$ were not obtained. Therefore, the IR transmittances were determined initially from only the first term on the righthand side of Eq. (10). Results of the comparison of the transmittances deduced by neglecting reflectance with cirrus cloud thicknesses are shown in Figs. 7a and $7 \mathrm{~b}$; in Fig. 7 a radiometric and lidar data refer to the same volume whereas data in $7 \mathrm{~b}$ refer to concurrent views of different volumes of the same cloud. The solid lines in Fig. 7 represent two-parameter least-squares fits to the formula

$$
\tau_{c}=\exp \{-a H[\exp (b H)]\},
$$

where $H$ is the cloud thickness. During the curve fitting, minimization of differences was achieved with respect to $\ln \left[-\ln \left(\tau_{c}\right) / H\right]$. The dashed lines are the result of least-squares fits in accordance with the simple linear relationship

$$
\tau_{c}=1-c H .
$$

In view of the anticipated scatter of data points, both relationships appear to yield good descriptions of the relationship between IR transmittance and cloud thickness. Eq. (11) yields a fit that is properly bounded in both extremes, whereas (12) is not properly bounded at the upper limit.

For many of the data points it was possible to perform an analysis to determine optical parameters from the lidar traces as described above. Thus, with the transmittance through the cloud at the ruby wavelength and in the infrared window region, it was possible to compare statistically the apparent extinction coefficients at the two wavelengths. Comparisons based on the preliminary analyses of transmittance indicated that the average ratio of the IR extinction coefficient to the extinction coefficient at the ruby wavelength was excessively large (beyond an acceptable upper limit of 2).

The experimental work of Burch et al. (1970) indicated that the ratio of extinction coefficients at 10.6 and $0.6328 \mu$ could be as large as 1.3. To examine further the probable magnitude of the ratio of extinction coefficients, some computations were made for "equivalent" ice spheres. For the same volume of monodisperse spherical particles, the ratio of the Mie extinction efficiencies will be the same as the ratio of the extinction coefficients. By using the approximate method described by Deirmendjian (1969) and real refractive indices close to 1.32 , the extinction coefficient at the 
ruby wavelength for spheres with radii $>10 \mu$ was found to be 2.0 to the first decimal place. At IR wavelengths between 10 and $13 \mu$ (in steps of $0.5 \mu$ ), the refractive indices listed by Irvine and Pollack ${ }^{4}$ (1968) were used to compute the extinction efficiencies for ice spheres with radii between 10 and $60 \mu$, thereby extending their tables. Table 2 is a condensed summary of the computed extinction efficiency factors throughout the infrared "window" region. If the refractive indices are correct and if the concept of an equivalent ice sphere is applicable, the results suggest that spectral measurements of transmittance might aid in the evaluation of an appropriate equivalent radius.

Fig. 8 is a plot of one-half of the ratio of the extinction efficiency at $10.5 \mu$, which is equivalent to the ratio of the extinction coefficients at 10.5 and $0.6943 \mu$, and should correspond approximately to the observations of Burch et al. (1970). It can be seen from Fig. 8 that only for equivalent spheres with radii between 14 and $20 \mu$ does the ratio exceed 1.3 , whereas for radii $\gtrsim 30$ microns a ratio of about 1.1 prevails.

Adjustments of preliminary analyses, without alteration of original calibration data, were required in order to obtain IR extinction coefficients that averaged less than 1.1 times larger than extinction coefficients for the ruby wavelength. Preliminary transmittances that were determined from the lidar data were decreased by reanalyzing the lidar traces (as illustrated in Fig. 6) for the maximum values of $\beta_{\pi}(R)$ and $b$ that were still compatible with the observations. Infrared transmittances were increased by including estimates for the reflectance term, the second term on the right-hand side of (10). Computation of IR fluxes and radiances indicated that, for a tropical atmosphere with high cirrus

TABLE 2. Refractive indices and computed extinction efficiency factors at several infrared wavelengths for ice spheres with radii between 10 and $60 \mu$.

\begin{tabular}{|c|c|c|c|c|c|c|}
\hline \multicolumn{7}{|c|}{ Refractive indices } \\
\hline & $\begin{array}{l}10.0 \\
10.5 \\
11.0 \\
11.5 \\
12.0 \\
12.5\end{array}$ & & & \multicolumn{3}{|c|}{$\begin{array}{l}1.152-0.0413 i \\
1.195-0.0602 i \\
1.290-0.0954 i \\
1.393-0.1135 i \\
1.480-0.1203 i \\
1.565-0.1184 i\end{array}$} \\
\hline \multicolumn{7}{|c|}{ Extinction efficiency factors } \\
\hline $\begin{array}{c}\text { Radius } \\
(\mu)\end{array}$ & 10.0 & 10.5 & $\begin{array}{l}\text { Wavele } \\
11.0\end{array}$ & $\begin{array}{c}\text { ngth }(\mu) \\
11.5\end{array}$ & 12.0 & 12.5 \\
\hline $\begin{array}{l}10 \\
(14) \\
20 \\
30 \\
40 \\
50 \\
60\end{array}$ & $\begin{array}{l}1.759 \\
(2.388) \\
2.748 \\
2.408 \\
2.165 \\
2.197 \\
2.188\end{array}$ & $\begin{array}{l}2.189 \\
(2.674) \\
2.661 \\
2.245 \\
2.223 \\
2.205 \\
2.167\end{array}$ & $\begin{array}{c}2.790 \\
(2.756) \\
2.363 \\
2.288 \\
2.228 \\
2.198 \\
2.175\end{array}$ & $\begin{array}{c}3.016 \\
(2.567) \\
2.345 \\
2.290 \\
2.242 \\
2.206 \\
2.183\end{array}$ & $\begin{array}{l}3.001 \\
(2.431) \\
2.423 \\
2.297 \\
2.248 \\
2.215 \\
2.191\end{array}$ & $\begin{array}{c}2.893 \\
(2.385) \\
2.459 \\
2.322 \\
2.259 \\
2.222 \\
2.197\end{array}$ \\
\hline
\end{tabular}

${ }^{4}$ A typographical error in their listing of the imaginary portion of the refractive index between 11.5 and $13.0 \mu$ was corrected.

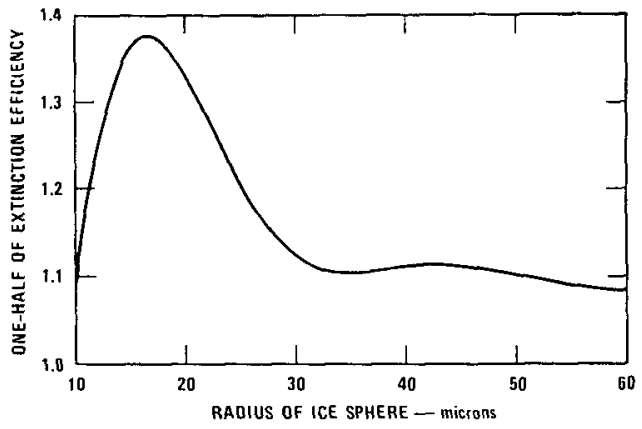

FIG. 8. Ratio of the extinction efficiencies at $10.5 \mu$ to those at $0.6943 \mu$ for ice spheres.

cloud above a warm ocean surface, the equivalent isotropic upward radiance from the sea surface could be as much as four times larger than the downward Planckian radiance at the cloud temperature. With sufficiently thick cirrus, a maximum reflectance parameter of 0.04 was postulated for $\rho_{c}$; this leads to a maximum increase of 0.11 in the deduced transmittance. For the thinnest clouds, this incremental increase of the initial transmittance must diminish toward zero. Thus, for example, the adopted correction was set at 0.03 , 0.075 and 0.10 for initial IR transmittances (neglecting reflectance) of $0.9,0.75$ and 0.6 , respectively, and was held constant at 0.11 for all initial transmittances $<0.45$.

Restricting data only to upward views and combining the data points shown in Fig. 7 allowed the determination of new relationships between cirrus thickness and the corrected IR transmittance. Results of the leastsquares fits according to Eqs. (11) and (12) are illustrated in the upper part of Fig. 9. It may appear that the curve of "best fit" is yielding underestimates of the transmittances for the thickest observed clouds. However, some of the transmittances have been overcorrected (shifted too far to the right) because the reflectance term actually is negligible for measurements taken within thick clouds. Therefore, the solid line is thought to be reasonable and is replotted in linear coordinates in the lower part of Fig. 9. It is this curve that is to be checked by future measurements and analyses for other cirrus clouds.

The statistical fit indicates that the infrared transmittance tends to vary linearly with cirrus thickness over a substantial range of thicknesses. This unexpected result is independent of assumptions inherent in the quantitative solution of the lidar equation and requires only an analysis of cloud thickness from the lidar data. Improvements in the quantitative analyses of both lidar and radiometric data are required before an empirical relationship (cf. Platt and Gambling, 1971) between the optical thicknesses at 0.6943 and $10-12 \mu$ can be established from a sizeable data set without excessive scatter. 

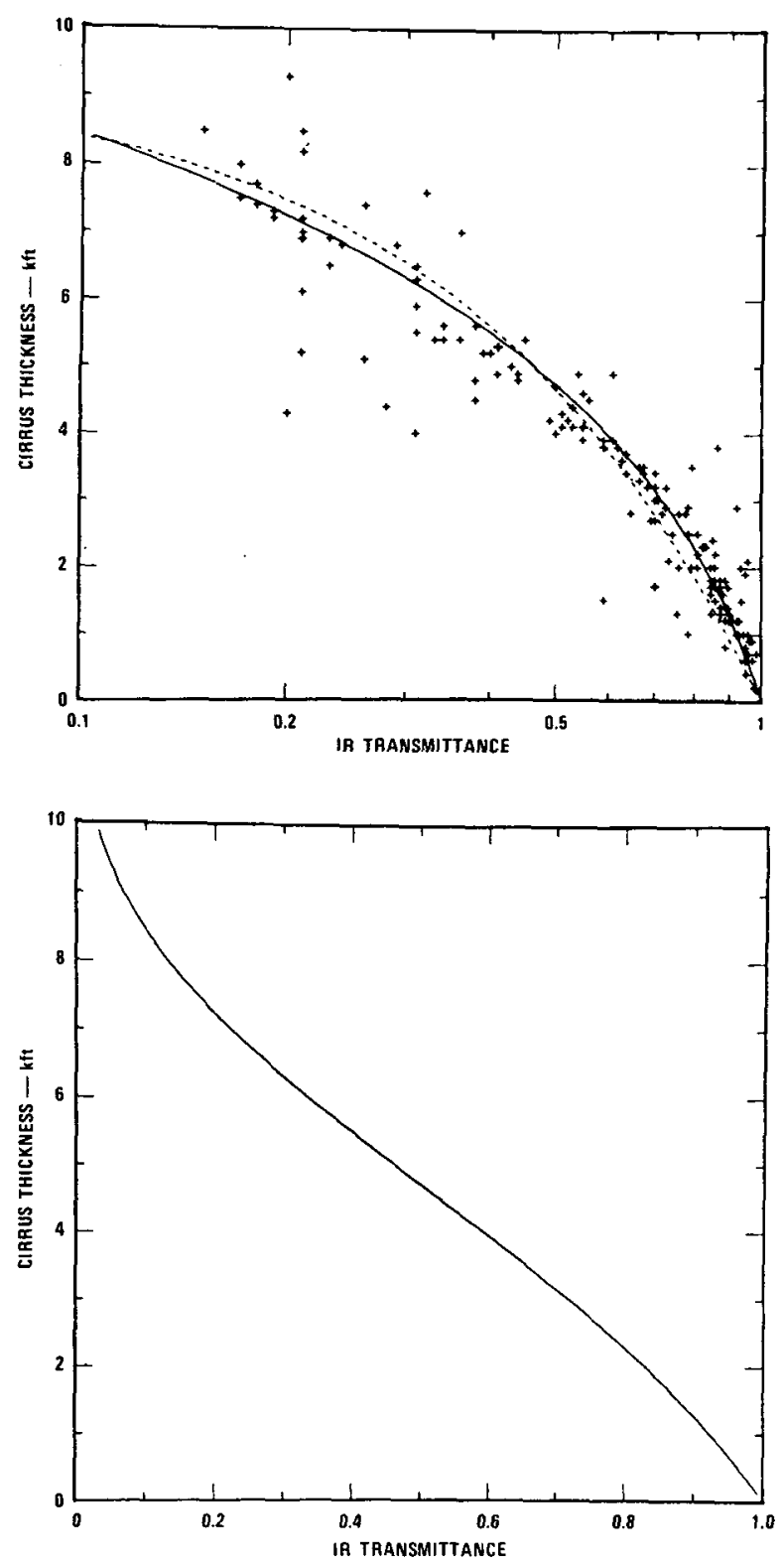

FIG. 9. Infrared transmittance $(10-12 \mu)$, corrected for probable reflectance, and the geometrical thickness of cirrus clouds. The semilog (upper) and linear (lower) plots with solid curves are from $\mathrm{Eq}$. (11); the dashed curve is from (12).

\section{Concluding remarks}

The analyses of concurrent airborne lidar and radiometric data from cirrus clouds indicate that a relatively simple statistical relationship exists between the infrared transmittance and the geometrical thickness of the cirrus cloud. The unique detection and ranging capabilities of the lidar system thus make it an almost indispensable component of experimental programs that are conducted to determine the influence of cirrus or haze on radiometric measurements. Furthermore, quantitative analyses of the lidar traces provide a means for objectively describing variations in the optical parameters (at the laser wavelength) of particulate layers. Subsequent comparison of optical properties at different wavelengths then permits an improved evaluation of the reliability of results.

As for the future, the study of the relationship between cirrus transmittance and cirrus thickness should be extended to other parts of the spectrum, both for solar and terrestrial radiation. In addition to improvements in techniques for data acquisition and processing, the airborne lidar system should incorporate a higher pulse-repetition frequency than that employed in this study. The higher pulse frequency will permit an averaging of data to reduce inherent scatter (especially from the assumed relationship between extinction and backscattering) in deduced optical properties, and, at the same time, will provide a better definition of the viewed background and the occurrence of holes in the cloud cover.

Acknowledgments. Mr. William Evans of SRI was primarily responsible for the successful preparation and operation of the ruby lidar aboard the aircraft. Dr. Peter Kuhn of the Environmental Research Laboratories, NOAA, conducted the concurrent radiometric measurements and provided the analyzed IR transmittances used in this report. Dr. William Marlatt and Mr. Harold Cole of Colorado State University furnished particle-count data collected aboard the aircraft. Dr. Edward Uthe of SRI performed the Mie computations used in the analysis. Considerable assistance in the overall experimental phases of the study was provided by Dr. Michal Bader and Mr. Earl Peterson of the NASA Ames Research Center and by Dr. William Nordberg and Mr. William Bandeen of the Goddard Space Flight Center, NASA.

\section{APPENDIX}

\section{Computations of Backscattering Coefficients}

Vertical profiles of the volume backscattering coefficient can be obtained in a relative sense from lidar measurements. To assign absolute magnitudes to profiles of the clear air backscattering coefficient (required for quantitative analysis of the lidar traces), it was necessary to compute the coefficients on the basis of independent, concurrently measured particle size distributions at points on the profile. Fig. 10 illustrates two of the size distributions that were measured by Colorado State University from aboard the NASA CV-990 aircraft over the BOMEX area. The distribution at an altitude of 700 refers to the humid subcloud layer, whereas that at $7000 \mathrm{ft}$ occurs beneath the typical height of the trade-wind inversion.

For some specified range of particle radius $a$, the particle concentration per radius interval was described in the form

$$
n_{a}=c a^{-(k+1)} \text {, }
$$




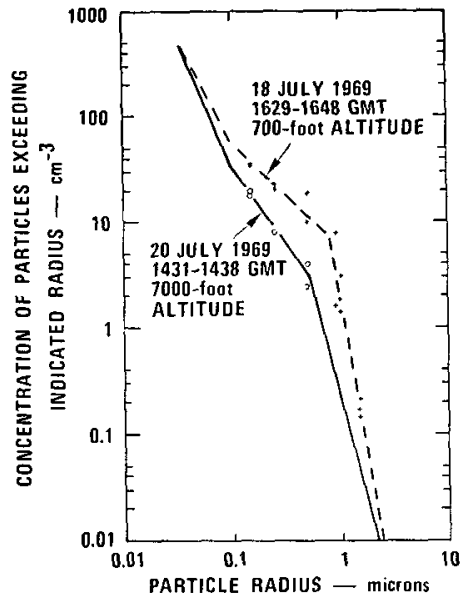

FIg. 10. Two particle size distributions from airborne observations by Colorado State University over the BOMEX area.

and the ordinate in Fig. 10 was defined by

$$
N_{a}=\int_{a}^{\infty} n_{a} d a
$$

As a matter of convenience, the straight line segments describing the size distributions in Fig. 10 were drawn with the constraint that the slope $k$ of each segment (representing a range of particle size) was a multiple of one-half. The intercept at $a=1$ of each segment is the logarithm of the ratio $c / k$ for that range of particle sizes, and the combination of segments represents a series of Junge-type distributions.

The total backscattering coefficient $\beta_{\pi}$ includes the sum of coefficients for particulate backscattering $\beta_{\pi}(P)$ and molecular backscattering $\beta_{\pi}(M)$. The particulate backscattering coefficient was computed for a range of refractive indices from

$$
\beta_{\pi}(P)=\sum\left[\pi c(2 \pi / \lambda)^{k-2} \int\left(q_{\pi} / x^{k-1}\right) d x\right]
$$

where $x$ is the size parameter $(2 \pi a / \lambda), \lambda$ the wavelength, $q_{\pi}$ is Mie angular scattering efficiency, and the summation is carried over the line segments used to describe the size distribution. The molecular backscattering coefficient $\left(\mathrm{km}^{-1} \mathrm{sr}^{-1}\right)$ at the ruby wavelength was computed from

$$
\beta_{\pi}(M)=(1.52)\left(10^{-4}\right) P / T,
$$

where $P$ is the pressure in millibars and $T$ the temperature in degrees Kelvin.

Ratios were formed of the computed total backscattering coefficients at the lower altitudes to the computed molecular backscattering at 17,000 ft. These ratios were compared to ratios derived from lidar measurements in the clear air at the same altitudes. The comparison indicated that:

1) Best agreement with the lidar data was obtained with a refractive index of $\sim 1.5$ above the moist subcloud layer and $\lesssim 1.45$ in the moist subcloud layer (see, for example, Eiden, 1966).

2) The total backscattering coefficient at $17,000 \mathrm{ft}$ was close to the molecular backscattering coefficient, or not more than about $25 \%$ larger.

\section{REFERENCES}

Burch, D. E., J. D. Pembrook and E. Reisman, 1970: Attenuation measurements in artificial clouds. Final Tech. Rept., Contract NAS 12-2124, Philco-Ford Corporation, Aeronutronic Division, Newport Beach, Calif.

Davis, P. A., 1969: The analysis of lidar signatures of cirrus clouds. Appl. Opt., 8, 2099-2102.

—-, 1970: Airborne ruby-lidar and radiometric measurements of cirrus and haze during BOMEX. Final Rept., Contract E-263-68, SRI Project 7334, Standord Research Institute, Menlo Park, Calif.

Deirmendjian, D., 1969: Electromagnetic Scattering on Spherical Polydispersions. New York, Elsevier, 290 pp.

Eiden, R., 1966: The elliptical polarization of light scattered by a volume of atmospheric air. Appl. Opt., 5, 569-575.

Irvine, W. M., and J. B. Pollack, 1968: Infrared optical properties of water and ice spheres. Icarus, 8, 324-360.

Johnson, W. B., and E. E. Uthe, 1971: Lidar observations of the lower troposphere during BOMEX. Boundary-Layer Meleor., 1 (in press).

Kuhn, P. M., 1970: Airborne observations of contrail effects on the thermal radiation budget. $J$. Atmos. Sci., 27, 937-942.

Platt, C. M. R., and D. J. Gambling, 1971: Emissivity of high layer clouds by combined lidar and radiometric techniques. Quart. J. Roy. Meteor. Soc., 97, 322-325.

Viezee, W., R. T. H. Collis and J. Oblanas, 1971: Lidar observations in relation to atmospheric winds aloft. J. Appl. Meteor., 10, $916-920$. 